\title{
Dynamic Response and Reliability Analysis of Buried Steel Pipes Under Blast Load
}

\author{
Shengwu TU ${ }^{\mathrm{a}, \mathrm{b}}$, Dongwang ZHONG ${ }^{\mathrm{a}, \mathrm{b}, 1}$, Linna LI ${ }^{\mathrm{a}, \mathrm{b}}$, Xiangchao GONG ${ }^{\mathrm{a}, \mathrm{b}}$ \\ and Kuan $\mathrm{ZHU}^{\mathrm{a}, \mathrm{b}}$ \\ ${ }^{a}$ College of Science, Wuhan University of Science and Technology, Wuhan, China \\ ${ }^{\mathrm{b}}$ Hubei Province Intelligent Blasting Engineering Technology Research Center, \\ Wuhan, China
}

\begin{abstract}
Comprehensively considering the influence of multiple load factors involved in the stress of buried pipelines, the dynamic response model and failure function of buried pressurized pipelines under explosive loads are established, and the formula for calculating the stresses of buried pressurized pipelines is proposed. Design and carry out dynamic response model experiment of buried steel pipe under explosive load, and extract relevant model parameters according to experimental data. For the buried pressurized steel pipes under extreme experimental conditions, the reliability analysis was carried out by Monte Carlo method, and the limit proportional distance of pipeline failure under experimental conditions was obtained. To determine the degree of influence of various parameter changes on the reliability of buried steel pipes. The research results can provide a certain reference for the quantitative risk assessment and safe operation analysis of the adjacent buried pipelines in blasting construction.
\end{abstract}

Keywords. Blasting load, buried pipeline, dynamic response, reliability analysis

\section{Introduction}

Explosive impact load is one of the main forms of third-party damage to pipelines, and the damage to buried pipelines may sometimes be irreparable. In the practice of explosive engineering, the explosion construction area is often adjacent to the in-service buried pipeline. On the basis of a good explosion effect, while ensuring the safety of adjacent buried pipelines is an issue of great concern to the explosion engineering industry [1].

For the calculation of pipeline failure probability, there are currently three main calculation models at home and abroad [2]:

(1) Database analysis model. Through the collection, statistics and analysis of failure information to determine failure factors, European and American countries have established relatively complete databases [3], but the current domestic pipeline failure database is not perfect, and it is difficult to effectively evaluate the failure probability of the pipeline system;

1 Dongwang Zhong, College of Science, Wuhan University of Science and Technology, Wuhan, China; E-mail: zhongdw123@wust.edu.cn. 
(2) Failure Tree analysis model. Zhang et al. [4] qualitatively analyzed the probability of pipeline failure events by establishing a fault tree; Wang et al. [5] used the fuzzy comprehensive evaluation method to evaluate the risk of oil and gas pipelines. However, it is difficult to objectively determine the weight with the fault tree model, and the result of the conditional independence test is unreliable;

(3) Theoretical model of structural reliability. It is the most effective and accurate to use the structural reliability model to calculate the failure probability when the failure database is not perfect. At present, the first-order second-moment method and Monte Carlo method are the most commonly used. Rackwita and Fiessler [6] performed reliability analysis on the pipeline based on the improved first-order second moment; Zhang et al. [7] used Monte Carlo method to study the effect of excavation on pipeline reliability during third-party damage quantitative impact.

Because the buried pipeline is a long-life and high-reliability structure, there are some problems, such as small number of failure samples under laboratory conditions, difficult to detect in the process of use, limited accumulation of information, difficult to obtain statistical rules and so on. In view of this, taking the buried pressurized steel pipe under explosive load as the research object, the dynamic response model and failure function are established, and the formula for calculating the stress of buried pressurized pipeline is put forward. the dynamic response model experiment of adjacent buried steel pipe under explosive load is designed and carried out, and the relevant parameters of the model are extracted according to the experimental data. Aiming at the buried pressurized steel pipe under the limit experimental condition, the reliability analysis is carried out by using the Monte Carlo method by using Matlab software, and the sensitivity analysis of the main parameters involved in the model is carried out to determine the influence degree of each parameter change on the reliability of buried steel pipe in the process of explosion experiment, in order to provide some data support for the quantitative risk assessment and safe operation analysis of buried pipeline in blasting construction.

\section{Dynamic Response Model and Failure Model of Buried Pipelines under Explosive Load}

\subsection{Methodological Assumptions}

The calculation method used in this paper is mainly used to predict the dynamic response of directly buried steel pipes under explosive load, analyze the pipeline strain caused by the explosive load, and combine with the thin shell theory to make the following assumptions: (1) The soil is uniform Qualitative and isotropic elastomer; (2) The pipeline is regarded as a thin-walled cylinder, and the pipeline is assumed to be a pressurizable empty pipe; (3) When the explosion load acts, the buried pipeline does not slide relative to the surrounding soil, that is, the pipeline The axial strain of is equal to the axial strain of the soil.

\subsection{Stress Model}

Buried pipelines produce axial stress, hoop stress and radial stress under various loads. For steel pipes, it can be regarded as a thin-walled cylinder. The axial, hoop and radial strains of the pipe develop along the axis and the periphery. When the pipe wall 
thickness is small compared with the diameter, the radial stress can be ignored. The following main research The axial and hoop stress of the pipeline. The axial and hoop stresses of buried steel pipes under explosive load are mainly caused by explosive impact load, pipeline internal pressure, temperature change and other reasons.

\subsubsection{Pipeline Stress Caused by Explosion Impact Load}

The formula for calculating the peak pressure of the particle in the free field under the impact of explosive ground in the soil medium is as follows:

$$
P_{0}=f \cdot\left(\rho_{c}\right) \cdot 160 \cdot\left(\frac{R}{W^{1 / 3}}\right)^{-n}
$$

Where $P_{0}$ is pressure. $f$ is the explosive ground shock coupling coefficient. $\rho_{c}$ is the acoustic impedances. $R$ is the distance between the mass point and the burst center in the free field. $W$ is the weight of the loaded explosive. $n$ is the attenuation coefficient.

In the above formula, the parameters such as $f, \rho_{c}$ and $n$ need to be obtained based on the results of field experiments. When the parameters are unknown, the axial stress of the explosion load on the pipeline can be simplified as:

$$
\sigma_{1}=k(\bar{R})^{\alpha}
$$

Where $\bar{R}$ is the proportional distance, taking into account the influence of the dose and the burst distance. $k$ is the site coefficient, a parameter reflecting the comprehensive influence of factors such as site, charge, pipeline, etc. $\alpha$ is the attenuation coefficient.

Kouretzis et al. [8-9] used a 3D thin-walled cylinder model to simplify the explosion wave into a harmonic load, deduced the calculation formula of the buried pipeline strain under the surface explosion load, and proposed a simplified calculation formula. Hao et al. [10] used the above method to combine the relationship between pipeline strain and vibration velocity and gave a concise expression of pipeline axial strain and hoop strain under explosive load. They believed that there is a certain proportional relationship between the two, which can be used for reference. The pipe hoop stress caused by the explosion impact load can be expressed by the following formula:

$$
\sigma_{2}=k_{1} \cdot \sigma_{1}=k_{1} k(\bar{R})^{\alpha}
$$

where $k_{1}$ is the proportional coefficient. Other parameters are the same as above.

The above formulas are all equations in the form of Lei Sa-style formulas, and their rationality is tested by experimental data. The parameters involved in $k_{1}, k$ and $\alpha$ etc. need to be extracted through experimental results.

\subsubsection{Pipeline Stress Caused by Internal Pressure and Temperature Changes}

According to the pipeline's strength check regulations in the "Oil Pipeline Engineering Design Specification GB50253-2014", the axial stress of the pipeline caused by the internal pressure and temperature changes of the pipeline is calculated as follows:

$$
\sigma_{3}=E \beta \Delta t+\mu \sigma_{4}
$$


where $E$ is the elastic modulus of the pipe. $\beta$ is the linear expansion coefficient of the material. $\Delta t$ is the difference between the installation temperature and the working temperature of the pipeline. $\mu$ is poisson's ratio. $\sigma_{4}$ is the circumferential stress generated by the internal pressure of the pipe.

The pipe is regarded as a thin-walled cylinder, and the hoop stress caused by the internal pressure of the pipe is calculated by Barlow's formula:

$$
\sigma_{4}=\frac{P D}{2 \delta}
$$

where $\mathrm{P}$ is the internal pressure of the pipe. $\mathrm{D}$ is the outer diameter of the pipe. $\delta$ is the wall thickness of the pipe.

In summary, the calculation formulas for the axial stress and hoop stress of the buried pressurized pipeline under explosive load can be obtained as:

$$
\begin{gathered}
\sigma_{\theta}=\sigma_{1}+\sigma_{3}=k(\bar{R})^{\alpha}+E \beta \Delta t+\mu \frac{P D}{2 \delta} \\
\sigma_{h}=\sigma_{2}+\sigma_{4}=k_{1} k(\bar{R})^{\alpha}+\frac{P D}{2 \delta}
\end{gathered}
$$

\subsection{Failure Function}

For buried pressurized pipelines under explosive load, the failure of the pipeline complies with the material's von Mises yield criterion, and the equivalent stress generated by the pipeline load can be expressed by the following formula:

$$
\sigma_{e q u l}=\sqrt{\sigma_{\theta}^{2}+\sigma_{h}^{2}-\sigma_{\theta} \sigma_{h}}
$$

where $\sigma_{\text {equl }}$ is the equivalent stress generated by the load on the pipeline. $\sigma_{\theta}$ is the axial stress generated by the load on the pipeline. $\sigma_{h}$ is the hoop stress generated by the load on the pipeline.

According to the "Code for Design of Oil Pipeline Engineering GB50253-2014", the allowable stress of the pipeline is calculated as follows:

$$
[\sigma]=K \cdot \varphi \cdot \mathrm{ss}
$$

where $K$ is the design coefficient, which is taken according to the specification, and the general section outside the oil transportation station should be 0.72 . $\varphi$ is the weld coefficient, which is $1 ; \sigma_{s}$ is the yield strength of the pipeline.

According to the Mises strength theory, the failure function of the buried pipeline can be expressed as:

$$
G(X)=[\sigma]-\sigma_{e q u l}
$$

When $G(X)>0$, the pipeline can operate normally, when $G(X)<0$, the pipeline cannot work normally, when $G(X)=0$, the pipeline is in a critical state. 


\section{Dynamic Response Experiment of Buried Pipeline under Explosive Load}

\subsection{Experiment Overview}

The test site is located in the Hubei Province Intelligent Blasting Engineering Technology Research Center, and the soil medium in the explosive test area is mainly cohesive soil. Three sizes of $20 \#$ seamless carbon steel pipes commonly used in buried pipeline construction are selected for the experiment. The pipe size is shown in table 1, and the relevant physical and mechanical parameters are shown in table 2.

Table 1. Size of seamless carbon steel pipe.

\begin{tabular}{lllll}
\hline Pipe number & Pipe length $/ \mathrm{m}$ & $\mathrm{D} / \mathrm{mm}$ & $\mathrm{d} / \mathrm{mm}$ & $\delta / \mathrm{mm}$ \\
\hline S1 & 4.5 & 110 & 101.5 & 4.24 \\
S2 & 4.5 & 159 & 149.6 & 4.7 \\
S3 & 4.5 & 300 & 291.2 & 4.4 \\
\hline
\end{tabular}

Table 2. Physical and mechanical parameters of seamless carbon steel pipe.

\begin{tabular}{llllll}
\hline$\rho / \mathrm{kg} / \mathrm{m}^{3}$ & $\mathrm{E} / \mathrm{GPa}$ & $\mu$ & $\mathrm{ss} / \mathrm{MPa}$ & $\mathrm{sb} / \mathrm{MPa}$ & $\beta /{ }^{\circ} \mathrm{C}^{-1}$ \\
\hline 7850 & 210 & 0.3 & 245 & 410 & $1.2 \times 10-5$ \\
\hline
\end{tabular}

The pipeline is buried directly. The bottom of the pipeline is padded with a thin layer of fine sand to prevent settlement after the pipeline is laid. The original soil is used for backfilling and tamping between the pipeline and the explosion source. The pipeline is processed before being buried to meet the experimental requirements, such as: (1) Weld seamless steel pipe heads at both ends of the pipeline, and both are sealed and tested for air tightness; (2) Pressurized valves and valves are installed at both ends Air compression equipment can be connected to facilitate pressurization and pressure relief during the experiment; (3) Strain gauges are installed at the four measuring points on the outer wall of the pipeline, and they are waterproofed. The pipeline burying diagram is shown in figure 1 , and the locations of the pipelines and explosion sources are shown in figure 2. For more experimental procedures and results, see references [11-13].

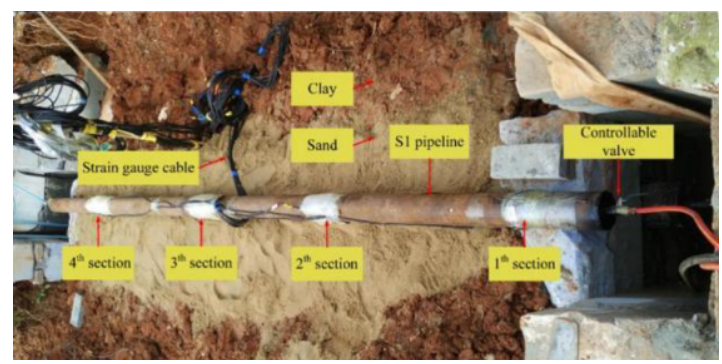

Figure 1. The buried pipe. 


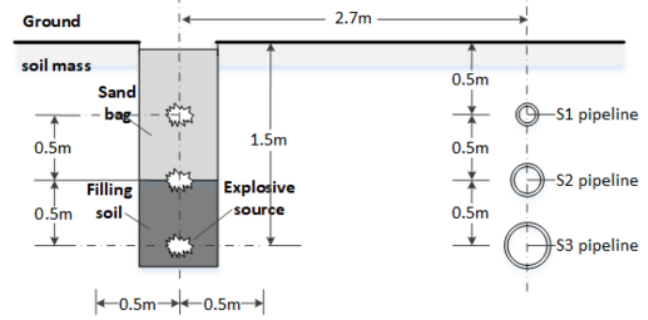

Figure 2. Schematic location of the pipes and the explosive source.

\subsection{Experimental Method}

In this experiment, BX120-3AA resistance strain gauge and UT3408 dynamic strain tester are used for strain measurement. Four cross sections are selected at the same interval of each pipeline, and each section is on the front, back, top, and bottom sides. 4 measuring points are arranged on the top, and each measuring point is arranged with two mutually perpendicular strain gauges, that is, strain gauges along the axial and circumferential directions.

The explosive source in this experiment uses $2 \#$ rock emulsion explosive, made into a spherical charge, and uses a coupled charge method. The explosive amount $\mathrm{W}$ is selected from $50 \mathrm{~g}, 75 \mathrm{~g}, 100 \mathrm{~g}, 125 \mathrm{~g}, 150 \mathrm{~g}, 175 \mathrm{~g}, 200 \mathrm{~g}$; For three locations, the explosive buried depth $\mathrm{H}$ is $0.5 \mathrm{~m}, 1 \mathrm{~m}, 1.5 \mathrm{~m}$, and the horizontal distance $\mathrm{D}$ from the pipeline is $2.7 \mathrm{~m}$, as shown in figure 2 . Before each explosion experiment, the pipeline is pressurized with air compression equipment. The value $\mathrm{P}$ is selected as $0 \mathrm{MPa}$, $0.2 \mathrm{MPa}, 0.4 \mathrm{MPa}, 0.6 \mathrm{MPa}$. After charging, the explosive chamber was blocked and covered, and one detonator was used to detonate, and the experimental results were recorded.

\subsection{Experimental Results and Analysis}

The parameters studied in this experiment include explosive charge $\mathrm{W}$, burst center distance $\mathrm{R}$ and pipeline internal pressure $\mathrm{P}$. Considering the need for reliability calculation in the following text, select the peak value of the upset surface of S3 pipe section 3 when the internal pressure $\mathrm{P}=0.6 \mathrm{MPa}$, the blasting source depth $\mathrm{H}=1.5 \mathrm{~m}$, and the burst center distance $\mathrm{R}=2.7 \mathrm{~m}$ under the extreme working conditions. Strain statistical results, experimental record results are shown in table 3.

Table 3. Experiment records.

\begin{tabular}{lllllllll}
\hline $\begin{array}{l}\text { Pipe } \\
\text { number }\end{array}$ & $\mathrm{W} / \mathrm{g}$ & $\mathrm{R} / \mathrm{m}$ & $\mathrm{P} / \mathrm{MPa}$ & $\mathrm{H} / \mathrm{m}$ & $\varepsilon_{1} / \mu \varepsilon$ & $\sigma_{1} / \mathrm{MPa}$ & $\varepsilon_{2} / \mu \varepsilon$ & $\sigma_{2} / \mathrm{MPa}$ \\
\hline & 50 & & & & 44.818 & 9.412 & 22.409 & 4.706 \\
& 75 & & & & 72.219 & 15.166 & 30.332 & 6.370 \\
$\mathrm{~S} 3$ & 100 & & & & 73.356 & 15.405 & 34.477 & 7.240 \\
& 125 & 2.7 & 0.6 & \multirow{2}{*}{1.5} & 115.979 & 24.356 & 55.670 & 11.691 \\
& 150 & & & & 120.963 & 25.402 & 52.014 & 10.923 \\
& 175 & & & & 129.175 & 27.127 & 58.129 & 12.207 \\
& 200 & & & & 142.403 & 29.904 & $74.049-$ & 15.550 \\
\hline
\end{tabular}

According to the experimental data, the relevant parameters under the 
experimental conditions $k_{1}=0.492 、 k=1096.66 、 \alpha=-2.337$, are obtained through the fitting formula, as shown in figure 3 and figure 4. From equation (2), the functional relationship between the proportional distance $\bar{R}$ and the axial peak stress $\sigma_{1}$ of the explosive load is obtained: $\sigma_{1}=1096.66 \times(\bar{R})^{-2.337}$, the correlation coefficient $R^{2}=0.945$, reliability Higher. The functional relationship between the proportional distance $\bar{R}$ and the hoop peak stress $\sigma_{2}: \sigma_{2}=539.56 \times(\bar{R})^{-2.337}$.

Under the condition that the pipeline installation temperature is $15^{\circ} \mathrm{C}$ and the working temperature is $25^{\circ} \mathrm{C}$, the remaining parameters of the pipeline can be obtained from table 1 and table 2. Substituting the above-mentioned $\sigma_{1}$ and $\sigma_{2}$ into the failure function of the pipeline, when the buried steel pipe is in In the critical failure state, that is, when $G(X)=[\sigma]-\sigma_{\text {equl }}=0$, the failure ratio distance under the experimental conditions $\bar{R}_{f}=2.217 \mathrm{~m} \cdot \mathrm{kg}^{-1 / 3}$.

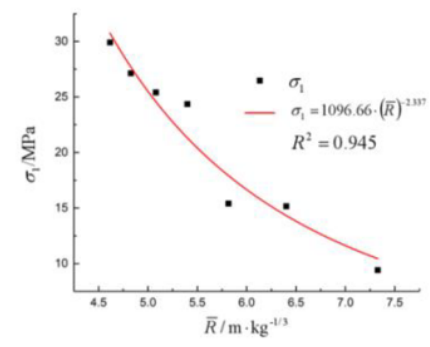

Figure 3. Fitting analysis of axial peak stress at of axial and hoop peak stress.

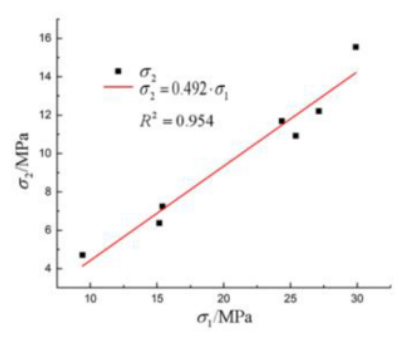

Figure 4. Fitting analysis of axial and hoop peak stress.

\section{Reliability Analysis Based on Monte Carlo Method}

\subsection{Reliability Calculation}

The above model is used to calculate the structural reliability of the S3 buried steel pipe under the failure ratio distance $\bar{R}=2.217 \mathrm{~m} \cdot \mathrm{kg}^{-1 / 3}$ in this experiment. The mean value and coefficient of variation of each parameter input into the model are reasonably selected with reference to the relevant literature, the pipeline itself and the experimental conditions. The relevant model constant parameters are shown in table 2 and the experimental fitting results. Although the statistical parameters may have a certain degree of correlation, at present, due to insufficient understanding of the relationship between the parameters, it is assumed that the random variables of the model are independent of each other. Based on the calculation example parameters, using Matlab software and Monte Carlo method to perform 106 random sampling simulations, the structural reliability of the S3 buried steel pipe is obtained as 0.4972 , which shows that the buried steel pipe at the failure ratio distance under the experimental conditions has a larger Probability of failure.

In order to further explore the limit proportional distance of the buried steel pipe under the experimental conditions, the proportional distance is increased from the above calculation example value to $125 \%$, and the other parameter calculation example values and the coefficient of variation remain unchanged, and the reliability of the buried steel pipe under the blasting load is analyzed. The change trend of the degree of 
pressure is shown in figure 5. Through the analysis of the change trend, the limit proportional distance of the buried steel pipe under the experimental conditions should be $\bar{R}_{r}=2.661 \mathrm{~m} \cdot \mathrm{kg}^{-1 / 3}$, which is that it does not cause pipeline failure. The minimum proportional distance.

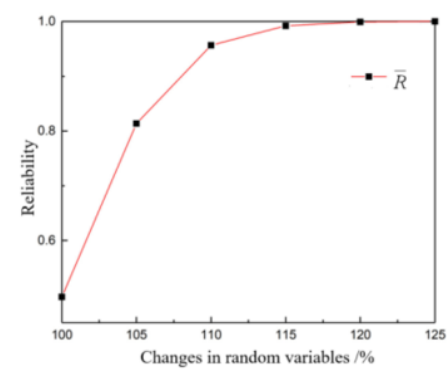

Figure 5. Impact of changes in proportional distance on reliability.

\subsection{Parameter Sensitivity Index Analysis}

The reliability of buried pipeline based on Monte Carlo method can be expressed as:

$$
\alpha_{i}=\frac{\Delta P_{r} / P_{r}}{\Delta C_{i} / C_{i}}
$$

where $C_{i}$ is the basic random variable; $\Delta C_{i}$ is the change of random variable; $P_{r}$ is the reliability of the structure; $\Delta P_{r}$ is the change in reliability caused by the corresponding $\Delta C_{i}$. The larger the $\alpha_{i}$ the greater the influence of the random variable $C_{i}$ on the reliability.

Based on the calculation of the reliability of the S3 buried steel pipe at the failure proportional distance above, all random variables except the proportional distance are increased from the example value to $125 \%$, and the coefficient of variation remains unchanged. The variation trend of the reliability of buried steel pipes when a certain random variable is changed while other random variables remain unchanged is shown in figure 6 , and the maximum sensitivity index of each parameter calculated by equation (11) is shown in figure 7. It can be seen that the proportional distance sensitivity index is the largest, followed by the temperature difference sensitivity index. The pipeline wall thickness, pipe outer diameter, pipe internal pressure and other parameter sensitivity indexes are close, that is, the change of the proportional distance has the greatest influence on the failure probability of the pipeline. Therefore, in the practice of blasting engineering, it is necessary to scientifically formulate a blasting plan based on site conditions, and strictly control the proportional distance, that is, the single shot charge and the blasting center distance, to ensure the safe operation of adjacent buried pipelines. In addition, the internal pressure of the pipeline should be appropriately reduced and increased. Large pipe wall thickness helps to improve the reliability of the pipe. 


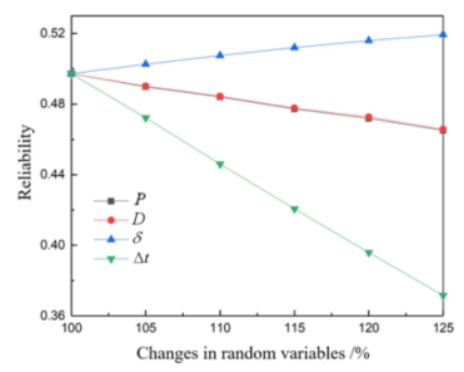

Figure 6. Impact of random variable changes on reliability.

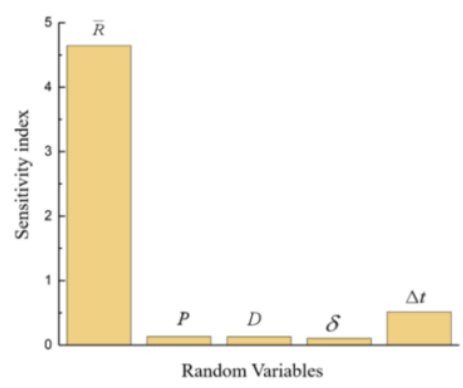

Figure 7. Maximum parameter sensitivity index.

\section{Conclusion}

(1) According to the pipeline strain monitoring data in the model experiment, the site coefficient $k$ and the attenuation index $\alpha$ were extracted from the model, and the calculation formula for the stress of the buried pressurized steel pipe under the explosive shock load under the conditions of the experimental worker's was obtained. According to the calculation of the failure function, the failure ratio distance of the pipeline under the model experiment condition is $2.217 \mathrm{~m} \cdot \mathrm{kg}^{-1 / 3}$.

(2) The Monte Carlo method is used to calculate the reliability of the buried pressurized steel pipe under the failure ratio distance, and the reliability of the buried steel pipe under this working condition is obtained as 0.4972 , and the limit ratio that does not cause pipeline failure is obtained through the reliability check The distance is $2.661 \mathrm{~m} \cdot \mathrm{kg}^{-1 / 3}$.

(3) Through parameter sensitivity analysis, it is found that the change of proportional distance has the greatest influence on pipeline failure probability. In blasting engineering practice, it is necessary to scientifically formulate a blasting plan according to site conditions, and strictly control the single shot charge and blasting center distance to ensure The operation of adjacent buried pipelines is safe.

\section{References}

[1] Liu X, Zheng S, Guo D, et al. Research progress of dynamic response of buried pipelines under the influence of blasting vibration. Pipeline Technique and Equipment. 2015Mar; 04: 48-54.

[2] Luo Z, Li J. Failure probability assessment of buried pipeline based on subset simulation. Journal of Safety Science and Technology. 2017 Sep; 13(3): 90-95.

[3] Bum JA, Young DJ. A method of quantitative risk assessment for transmission pipeline carrying natural gas. Journal of Hazardous Materials. 2005 Jan;123(1/3): 1-12.

[4] Zhang Z, Cheng Q, Sun W. Research of failure probability of congelation in oil pipeline based on FTA. Process Equipment \& Piping. 2015Jun; 52(5): 58-61.

[5] Wang C, Yang W, Yin H, et al. Application of fuzzy comprehensive evaluation in risk evaluation of pipeline crossing engineering. China Safety Science Journal. 2010Sep; 6(3): 96-100.

[6] Rackwita R, Fiessler B. Structural reliability under combined ranfom load sequences. Computers \& Structures. 1978 Aug; 9(5): 489-494.

[7] Zhang Q, Yang Y, Zheng H, et al. Study on reliability evaluation of pipeline under the effect of third party excavation. Journal of Safety Science and Technology. 2017 Nov; 13(2): 143-147.

[8] Kouretzis GP, Bouckovalas GD, Gantes CJ. Analytical calculation of blast-induced strains to buried pipelines. International Journal of Impact Engineering. 2007 May; 34(10): 1683-1704. 
[9] Kouretzis GP, Bouckovalas GD, Gantes CJ. 3-D shell analysis of cylindrical underground structures under seismic shear(S) wave action. Soil Dynamic and Earthquake Engineering. 2006 May; 26(10): 909-921.

[10] Hao Y, Cheng K, Zhao Q, et al. Study on safety distance of gas pipeline under action of blasting seismic wave. Blasting. 2018 Apr; 35(2): 137-143.

[11] Zhong D, Huang X, Si J, et al. Experimental Study on dynamic response of buried pipeline under blasting loads. Blasting. 2018 Mar; 35(2): 19-25.

[12] Zhong D, Huang X, Lu Z, et al. Experimental study on dynamic response of buried steel tubes with different sizes under blasting loading. Blasting. 2018 Jun; 18(13): 219-223.

[13] Gong X, Zhong D, Si J, et al. Dynamic responses of hollow steel pipes directly buried in high-saturated clay to blast waves. Explosion and Shock Waves. 2020 Feb; 40(02): 13-25. 